\title{
CORRIGENDUM
}

\section{Tunable and sizable band gap of single-layer graphene sandwiched between hexagonal boron nitride}

Ruge Quhe, Jiaxin Zheng, Guangfu Luo, Qihang Liu, Rui Qin, Jing Zhou, Dapeng Yu, Shigeru Nagase, Wai-Ning Mei, Zhengxiang Gao and Jing Lu

NPG Asia Materials (2012) 4, e16; doi:10.1038/am.2012.29; published online 27 April 2012

Correction to: NPG Asia Materials (2012) 4, e6; doi:10.1038/ am.2012.10; published online 17 February 2012

Since the publication of the above article, the authors have noticed inconsistency in the references to the 12 configurations presented in Figure 1. In the article text, numerical references (1-12) are given to those configurations, whereas they are labeled alphabetically (a to l) in Figure 1. 'Configuration 1-12' in the article text should correspond to Figure 1 (a)-(1), respectively. For example, 'Configuration 1' in the text refers to 'Figure 1 (a)' in the image.

The authors would like to apologize for the inconvenience. 